\title{
Directing the Aggregation of Native Polythiophene during in Situ Polymerization
}

\author{
Jenny Lebert, ${ }^{\dagger}$ Eva M. Kratzer, ${ }^{\dagger}$ Axel Bourdick, ${ }^{\dagger}$ Mihael Coric, ${ }^{\dagger}$ Stephan Gekle, ${ }^{\ddagger}$ and Eva M. Herzig ${ }^{*},, \S_{\odot}$ \\ ${ }^{\dagger}$ Herzig Group, Munich School of Engineering, Technische Universität München, Lichtenbergtstraße 4a, 85748 Garching, Germany \\ ${ }^{\ddagger}$ Biofluid Simulation and Modeling, Theoretische Physik, Universität Bayreuth, Universitätsstraße 30, 95440 Bayreuth, Germany \\ ${ }^{\S}$ Dynamics and Structure Formation - Herzig Group, Experimentalphysik, Universität Bayreuth, Universitätsstraße 30,95447 \\ Bayreuth, Germany
}

\section{Supporting Information}

ABSTRACT: The performance of semiconducting polymers strongly depends on their intra- and intermolecular electronic interactions. Therefore, the morphology and particularly crystallinity and crystal structure play a crucial role in enabling a sufficient overlap between the orbitals of neighboring polymers. A new solution-based in situ polymerization for the fabrication of native polythiophene thin films is presented, which exploits the film formation process to influence the polymer crystal structure in the resulting thin films. The synthesis of the insoluble polythiophene is based on an oxidative reaction in which the oxidizing agent, iron(III) p-toluenesulfonate (FeTos), initially oxidizes the monomers to enable the polymer chain growth and secondly the final polymers, thereby chemically doping the polythiophene. To exploit the fact that the doped polythiophene has a different crystal packing structure compared to the undoped polythiophene, we investigate the structural effect of this inherent

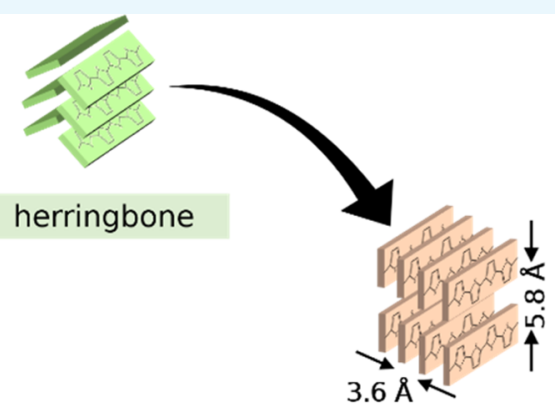

$\pi$-stacking doping process by varying the amounts of FeTos in the reaction mixture, creating polythiophene thin films with different degrees of doping. The structural investigation performed by means of grazing incidence wide-angle X-ray scattering (GIWAXS) suggests that the strongly doped polymer chains aggregate in a $\pi$-stacked manner in the film formation process. Moreover, this $\pi$-stacking can be maintained after the removal of the dopant molecules. GIWAXS measurements, molecular dynamics simulations, and spectroscopic analysis suggest the presence of polythiophene in a novel and stable crystal structure with an enhanced intermolecular interaction.

\section{INTRODUCTION}

Semiconducting polymers are the subject of intense research because of their highly attractive applications in organic electronic devices. ${ }^{1-3}$ The charge carrier transport in the conjugated polymers takes place either along the conjugated backbone of a single polymer chain (intramolecular) or through the overlapping orbitals of neighboring molecules (intermolecular). While the intramolecular transport requires an elongated and planarized backbone for an outstretched conjugated system, the intermolecular transport depends on the overlap of the orbitals and therefore on the geometry of the backbone aggregation, i.e., the crystal structure. Hence, the electronic properties of semiconducting polymers are strongly dictated by their morphology and crystallinity. ${ }^{4,5}$ Thin films of semiconducting polymers, however, are often amorphous or semicrystalline, creating a need for methods to enhance their crystallinity. Changing the molecular structure of the polymers is the most common approach to induce crystallinity. The addition of, for example, electronically insulating alkyl side chains to the backbone strongly increases the tendency of semiconducting polymers to crystallize, leading to a significant improvement of their electronic properties. Moreover, the side chains enhance the polymer solubility, enabling an easier processing from solution. One of the most investigated semiconducting polymers that was designed accordingly is the polythiophene (PT) derivative poly-3-hexylthiophene (P3HT).

In this letter, we present how crystallization and intermolecular interaction of polythiophene (PT) can be steered by the choice of processing route instead. We introduce a solution-based in situ polymerization which employs iron(III) p-toluenesulfonate hexahydrate (FeTos) as an oxidizing agent and a chemical dopant in the same fabrication step. The included doping process additionally has a strong effect on polymer aggregation, inducing a new and stable PT crystal structure that leads to an enhanced chromophore interaction within the semiconducting thin films. Since the dopant is rinsed out after the synthesis, no electronically inactive material remains incorporated in the insoluble thin films.

Received: April 9, 2018

Accepted: May 22, 2018

Published: June 13, 2018 


\section{RESULTS AND DISCUSSION}

We choose to perform the oxidative polymerization reaction with FeTos, as it is well-established in the in situ synthesis of high-performing poly-3,4-ethylenedioxythiophene (PEDOT) thin films. ${ }^{6}$ In our study, bithiophene (BT) serves as a monomer molecule for the polymerization of polythiophene. We perform the in situ polymerization from a metastable reaction mixture of both BT and FeTos. While within the dilute solution the reaction is prevented by the close redox potentials of the two reactants, the polymerization only starts during the processing, when the concentration of the reactants is significantly increased by the drying of the solvent. ${ }^{7,8}$ The reaction mechanism can be divided into two subsequent steps. ${ }^{9}$ Initially, iron(III) ions oxidize the monomer molecules to radical cations which subsequently polymerize. Depending on the employed amount of oxidizing agent, the already synthesized polymer chains are further oxidized by excess iron(III) ions in a second step. Effectively, this further oxidation is a chemical doping of the chains, introducing free charge carriers into the conjugated molecules. ${ }^{9}$ The positive charges on the chain are stabilized by the anions of FeTos, the tosylates, which are electrostatically attached to it. Simulations show that the tosylates align perpendicular to the polythiophene backbones. ${ }^{10} \mathrm{We}$ are interested in fabricating an insoluble semiconducting material with improved performance, exploiting the doping procedure during the processing. We therefore spin coat the metastable mixture and subsequently rinse out all residual monomers and oxidant molecules. It is known that the choice of washing solvent has a strong effect on the electronic properties of the obtained $\mathrm{PT} .{ }^{11}$ While rinsing in acetonitrile leaves the polymer chains in their doped and therefore in conducting state, ethanol additionally de-dopes the PT and removes the previously electrostatically attached tosylate counter ions, leaving PT in its semiconducting, unsubstituted state. Hence, we are able to obtain doped and undoped PT in the final thin films, depending on the last rinsing step, but using the same reaction mixtures. For an improved intermolecular interaction, the thin films of semiconducting PT are thermally annealed at $200{ }^{\circ} \mathrm{C}$ for $10 \mathrm{~min}$ right after the washing procedure.

To investigate the effect of the doping process occurring during the film formation step on the nanostructure of the final PT films, we vary the molar fraction of FeTos

$$
X_{\mathrm{FeTos}}=\frac{n_{\mathrm{FeTos}}}{n_{\mathrm{FeTos}}+n_{\mathrm{BT}}}
$$

with $n_{\mathrm{FeTos}}$ and $n_{\mathrm{BT}}$ as the amounts of FeTos and BT in the reaction mixture, respectively. We employ fractions ranging from 0.25 to 0.75 . Afterwards, we investigate the properties of both processing routes with conducting (acetonitrile-rinsed) and semiconducting (ethanol-rinsed and annealed) PT films separately. First, we analyze the electronic and structural properties of the conducting PT films by means of four-point probe measurements, UV/vis spectroscopy, and grazing incidence wide-angle X-ray scattering (GIWAXS). Thereafter, we show the effect of the process on the structure (GIWAXS) and the electronic interaction (UV/vis absorbance and emission spectroscopy) of the semiconducting PT chains.

We performed four-point probe measurements on the conducting PT films to show that the final doping level is controlled by the amount of FeTos in the reaction mixture. Figure 1a displays the in-plane electrical conductivities which a
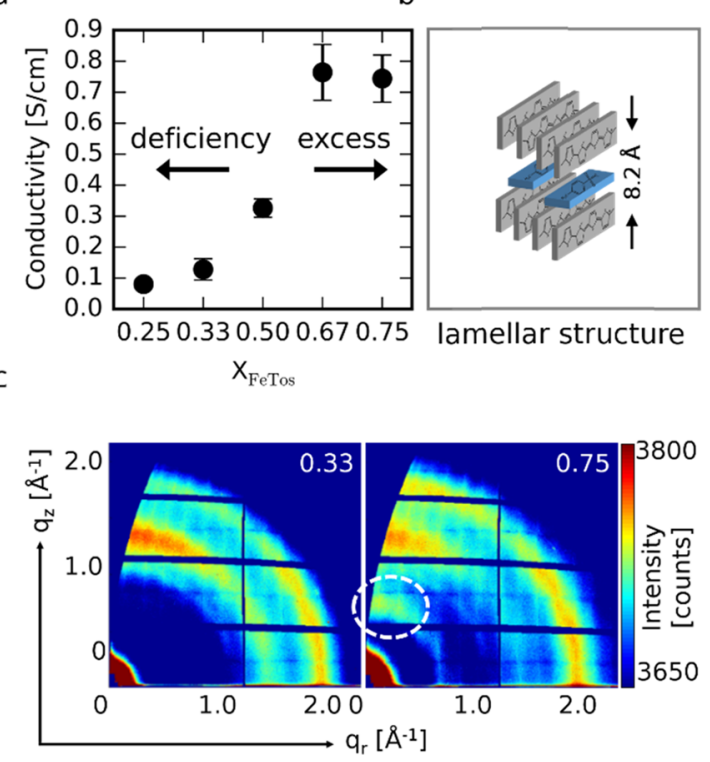

Figure 1. Properties of in situ polymerized conducting polythiophene thin films. (a) In-plane conductivities of films synthesized from the reaction mixtures with varied molar fractions of FeTos obtained by four-point probe measurements. (b) Schematic depiction of the proposed lamellar structure of doped PT and tosylate ions, and the given crystal lattice distance corresponds to lamellar backbone spacing, orientation of tosylate according to simulations found in the literature. $^{10}$ (c) The two-dimensional (2D) GIWAXS pattern of films synthesized with FeTos molar fractions of 0.33 (FeTos deficiency) and 0.75 (FeTos excess), Bragg diffraction of lamellar ordering is encircled in white.

we employ as a measure for the doping levels of the films. All conductivities are in the range of $0.1-0.8 \mathrm{~S} \mathrm{~cm}^{-1}$ and thereby several orders of magnitude above the conductivity of semiconducting, undoped PT $\left(10^{-8}-10^{-4} \mathrm{~S} \mathrm{~cm}^{-1}\right){ }^{12,13}$ Hence, rinsing in acetonitrile definitely leaves the PT thin films in their doped, conducting state. The achieved conductivities are of the same dimension as for the doped PT thin films synthesized in situ with other oxidizing agents.? Furthermore, the conductivity shows a drastic increase with increasing molar fraction $X_{\mathrm{FeTos}}$ in the reaction mixture.

While it is around $0.1 \mathrm{~S} \mathrm{~cm}^{-1}$ for low $X_{\mathrm{FeT} \text { os }}(0.25$ and 0.33$)$, it rises up to $0.8 \mathrm{~S} \mathrm{~cm}^{-1}$ beyond a fraction of 0.5 . Thus, we divide the investigated films into two different regimes. The films prepared from the reaction mixtures with $X_{\mathrm{FeTos}}$ below 0.5 are from now on referred to as synthesized under iron deficiency, as they show a strongly decreased doping level in comparison to those synthesized under iron excess $\left(X_{\mathrm{FeTos}}\right.$ larger than 0.5 ). This is additionally supported by the UV/vis absorbance spectra of the conducting thin films (see Figure S1), which show a strong rise in the absorbance band of the doped thiophene chromophores with respect to the $\pi-\pi^{*}$ absorbance band of the undoped molecules with increasing $X_{\mathrm{FeTo}}$, supporting that the increased conductivity is indeed caused by a higher fraction of oxidized thiophenes and not, for example, by a change in film quality or polymer chain length.

We investigate the structural effect of the doping level on the thin film morphology by performing GIWAXS measurements. Figure 1c shows the obtained $2 \mathrm{D}$ images of the conducting films obtained from the reaction mixtures with $X_{\mathrm{FeT} \text { Tos }}$ of 0.33 and 0.75 , respectively. Both scattering patterns exhibit two broad signals centered at $q=1.4$ and $1.8 \AA^{-1}$ (real space 
distances $d=4.5$ and $3.5 \AA$ ). These peaks are in agreement with what has been observed for doped, conducting PT synthesized via an oxidative polymerization e.g., as nanoparticles or in bulk. ${ }^{14-16}$ At a high doping level $\left(X_{\mathrm{FeTos}}=0.75\right)$, however, an additional Bragg signal arises at $q=0.77 \AA^{-1}(d=8.2 \AA)$, implying the development of an additional new crystal plane. Yamamoto et al. first found that the bulk PT changes its packing motif beyond a critical level of doping with iodine. ${ }^{16}$ According to their findings, a high dopant concentration results in a structural rearrangement of the molecules. The polythiophene backbones arrange in a $\pi$-stacked fashion, with the planar molecules parallel to each other. A lamellar structure evolves, in which the dopant molecules are located in between these PT $\pi$-stacks. Later, this effect has been explained by the tendency of oligothiophene cations to form $\pi$-stacked dimers. ${ }^{17,18}$ These dimers develop a chemical bond between the two parallel-arranged backbones, resulting in an additional stabilization of the positive charges. If a large number of positively charged oligothiophenes is present in a system, they even form $\pi$-stacked crystal structures. ${ }^{17,18}$ Chaalane et al. performed density functional theory calculations of tosylatedoped oligothiophenes to predict the orientation of the tosylate anions with respect to the polymer backbones. They found the sidewise arrangement to be most stable, with an intermolecular distance of $3.56 \AA$ between the sulfur atoms of polythiophene and tosylate, respectively. ${ }^{10}$ Therefore, we propose the formation of a similar crystal structure for the PT thin films synthesized under iron excess in our study. The highly doped PT chains arrange in a lamellar structure, giving rise to the new Bragg peak arising in the GIWAXS pattern for $X_{\mathrm{FeTos}}=0.75$. It is therefore assigned to a lamellar distance of $8.2 \AA$ between the polythiophene layers, with tosylate anions sandwiched in between as depicted in Figure $1 \mathrm{~b}$.

In the following, we discuss the structure and properties of the semiconducting PT films obtained from the same synthesis by subsequent rinsing with ethanol. Usually, semiconducting native PT is known to exhibit only a short-range order, with the backbones aggregating in a herringbone ( $\mathrm{HB}$ ) fashion as displayed in Figure 2d. The HB packing motif, induced by the electrostatic repulsion of the $\pi$-orbitals, leads to a poor overlap of the $\pi$-orbitals. Therefore, the intermolecular electronic interaction in unsubstituted PT is weak when compared to its substituted, $\pi$-stacked derivatives such as P3HT.

Figure 2a shows the 2D GIWAXS images we measured for semiconducting PT films synthesized under iron deficiency $\left(X_{\mathrm{FeTos}}=0.33\right.$, left image $)$ and strong iron excess $\left(X_{\mathrm{FeTos}}=0.75\right.$, right image), respectively. Both patterns show three sharp rings at scattering vectors $q=1.40,1.63$, and $1.98 \AA^{-1}$. While these rings are isotropic for $X_{\mathrm{FeT} \text { os }}=0.33$, meaning the crystallites have no favored orientation with respect to the sample surface, they show a strong anisotropy in the case of $X_{\mathrm{FeTos}}=0.75$. Here, the peak intensity is increased in a vertical direction. On the basis of the positions of the rings, we assign them to the main Bragg peaks of polythiophene HB crystals. ${ }^{16,19,20}$ Since these peaks are assigned to lattice distances perpendicular to the backbone axis, the anisotropy in the excess film is interpreted as $\mathrm{HB}$ crystallites with polymer backbones oriented preferably parallel to the substrate plane. ${ }^{12,21}$

For further analysis of the crystal structures, we radially integrate the intensities of the GIWAXS pattern in vertical (Figure 2b) and horizontal directions (Figure 2c). The vertical cuts of both samples show the three distinct peaks, which match the $\mathrm{HB}$ crystal structure. Additionally, a small peak arises a
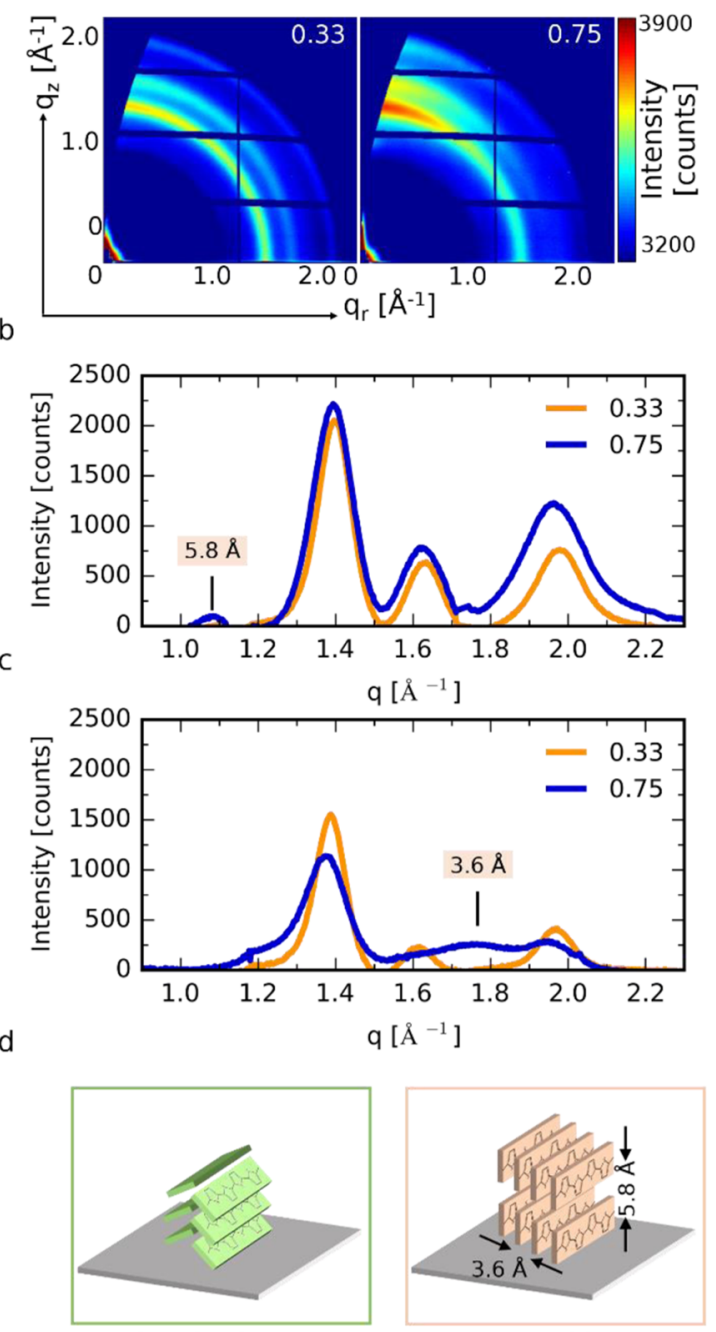

herringbone

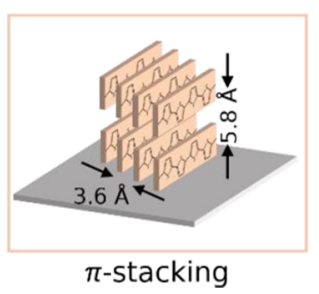

Figure 2. Properties of in situ polymerized semiconducting polythiophene thin films. (a) Two-dimensional GIWAXS scattering pattern of films obtained with FeTos molar fractions of 0.33 (FeTos deficiency) and 0.75 (FeTos excess). (b) Radially integrated scattering intensity in the vertical and (c) horizontal directions. The marked peaks correspond to the $\pi$-stacked crystal structure. (d) Schematic depiction of the two crystal structures of the semiconducting PT: herringbone (green box) and $\pi$-stacking (beige box).

at $q=1.08 \AA^{-1}(d=5.8 \AA)$ when iron excess is employed in the synthesis. In the horizontal cuts there is an even larger difference between the two samples. In the horizontal direction, the PT synthesized with $X_{\mathrm{FeTos}}=0.75$ exhibits a new peak at approximately $q=1.76 \AA^{-1}(d=3.6 \AA)$. From these two new peaks, we conclude that an additional crystal structure is present in the PT thin films for iron excess. On the one hand, the three most prominent peaks of both samples fit to the natural herringbone structure that is known for unsubstituted polythiophene. For PT synthesized under iron deficiency, this seems to be the only crystal structure present within the final semiconducting film. The second sample, prepared under iron excess, on the other hand, shows two additional peaks, one in each of the respective cuts, which is present after washing out the tosylate ions and annealing the film. From the examination of the conducting films, we know that the samples with iron excess contain $\pi$-stacked PT structures forming a lamellar 
structure with the tosylate ions. Since the processing prior to the washing is identical, we analogously propose a $\pi$-stacked structure for the semiconducting PT. The detected new lattice distance of $3.6 \AA$ is close to the calculated intermolecular distance for tosylate-doped oligothiophenes $(3.7 \AA)^{10}$ and also in the same range as the $\pi$-stacking distances of both P3HT $(3.8 \AA)^{22}$ and PEDOT $(3.4 \AA) .{ }^{23}$ The small peak arising in the vertical direction is consequently interpreted as the side-by-side distance between the backbones. Hence, the difference between the lamellar spacing in the conducting PT processed with iron excess $(8.2 \AA)$ and the backbone distance in the semiconducting PT processed with iron excess (5.8 ̊) must arise from the removal of the tosylate ion in the ethanol-washing process and a resulting shrinkage of the intermolecular distance. The proposed crystal structure is schematically displayed in Figure 2d.

To our knowledge, this is the first reported $\pi$-stacked crystal structure of undoped, semiconducting, unsubstituted polythiophene. The GIWAXS data clearly show that in the presented processing route the doping of the polymer backbones during the film formation has a strong effect on the resulting PT thin film structure. Beyond a critical level of doping, the polymer chains rearrange into a lamellar structure of alternating cationic PT $\pi$-stacks and layers of tosylate counter ions in between. However, contrary to the findings of Yamamoto et al. for bulk PT, the induced $\pi$-stacking stays intact after the tosylate is removed by rinsing with ethanol. The $\pi$-stacked crystal structure is maintained even after thermal annealing at $200{ }^{\circ} \mathrm{C}$, indicating a certain stability. Using molecular dynamics (MD) simulations, we can confirm the feasibility of the discussed structures of PT without the presence of tosylate. We simulate a crystal of 100 PT 10-mers. The calculations show that if the PT chains are constrained to the crystal plane separations observed in the experiment (5.8 and $3.6 \AA$ ), but are nevertheless free in rotation around the backbone, all the PT chains tilt in the same direction and form $\pi$-stacks. When removing the constraints, the vertical PT stacks slide in position and tilt in an alternating fashion, resulting in a typical herringbone structure with the calculated separations (unit cell parameters $a=7.10 \AA$ and $b=5.71 \AA$ ) agreeing well with the experimental values here and in the literature $(a=7.81$ $\AA$ and $b=5.56 \AA) .{ }^{19,20}$ This indicates that the herringbone structure exhibits a strong global minimum, while a $\pi$-stacked structure is possible if the movement of the polymer chains is prevented due to the polymer chains being embedded in the densely packed films (see Figure S2).

Finally, we perform absorbance and emission spectroscopy measurements on the semiconducting thin films to investigate the effect of the altered crystal structure on the intermolecular interaction of the PT chains. Figure 3 shows the spectra of the semiconducting PT films. The UV/vis absorbance of all investigated thin films expands from the ultraviolet region to beyond $600 \mathrm{~nm}$. As the plot shows, the maximum of the absorbance shifts from 465 to $500 \mathrm{~nm}$ for PT synthesized under iron excess. Moreover, the intensity decreases in the low wavelength region, while the intensity of the shoulders increases at higher wavelengths. We assume that the absorption properties of PT are very similar to those of the wellinvestigated $\mathrm{P} 3 \mathrm{HT}$, since their backbones are composed of the same thiophene chromophores. Therefore, we can interpret the spectra as a superposition of the amorphous contributions of the disordered PT chains in the higher energy section and the vibronic transitions of the aggregated thiophene chromophores

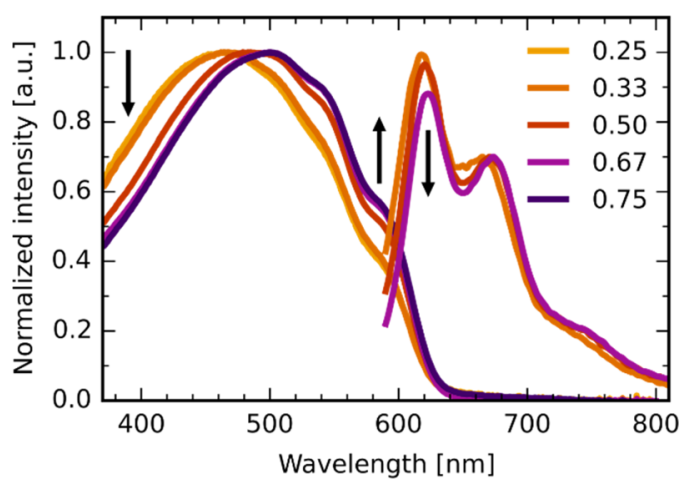

Figure 3. Normalized absorbance and emission spectra of semiconducting PT films polymerized in situ with varied molar fractions of FeTos (see legend). Each absorbance spectrum is normalized to its respective maximum, emission spectra are normalized to the intensity of the 0-1 transition. Arrows indicate a decrease in amorphous contributions and an increase of vibronic transitions in the absorbance spectra and a decrease of the $0-1$ transition in the emission spectra. Excitation wavelength for all emission measurements is $500 \mathrm{~nm}$.

of elongated, ordered polymer chains at higher wavelengths. ${ }^{24}$ Hence, the fraction of ordered PT backbones in the films increased when synthesized under iron excess.

Moreover, the relative increase of the vibronic shoulders compared to the amorphous contribution induces the shift of the absorbance maximum, although the positions of the single absorbance bands seem to remain unchanged. Thus, the chemical constitution of the semiconducting polymers is not altered by the different synthetic parameters.

The emission spectra give further insight into the character of the chromophore interaction. All spectra exhibit the most intense emission band at around $620 \mathrm{~nm}$ and a second strong emission at $670 \mathrm{~nm}$. With increasing fraction of FeTos, the relative intensity of the first band decreases. Moreover, the spectra are slightly redshifted at higher fractions. The energy of the different emissions as well as the ratio between the single emission bands is comparable to those of aggregated but not fully crystallized P3HT, with the thiophene chromophores surrounded by the $\pi$-system of the other backbones. ${ }^{24}$ This is plausible, as PT is the backbone of P3HT and therefore features very similar electronic properties. The decreasing ratio of the $0-0$ to $0-1$ emission is a clear indication of an increased order within the films. The redshift on the other hand suggests an increased interaction between the chromophores of neighboring polymer chains. Therefore, the synthesis under iron excess appears to have two effects: firstly, the overall order within the thin film increased, while secondly, the new $\pi$-stacked crystal structure results in an increased intermolecular interaction between the backbones compared to the purely $\mathrm{HB}$ packed PT. $^{25,26}$

Combining the results of the four-point probe measurements, the GIWAXS data and spectroscopic analysis, we draw a model of how the doping process influences the crystal structure of the in situ polymerized PT. Figure 4 schematically depicts the processes of the BT polymerization and the following doping as well as the resulting crystal structures of the differently doped chains. In the first part of the film forming process, the monomers are oxidized by the iron ions and subsequently polymerize. Although the oligothiophenes become insoluble beyond a certain chain length, they still possess enough mobility to undergo additional oxidation by 

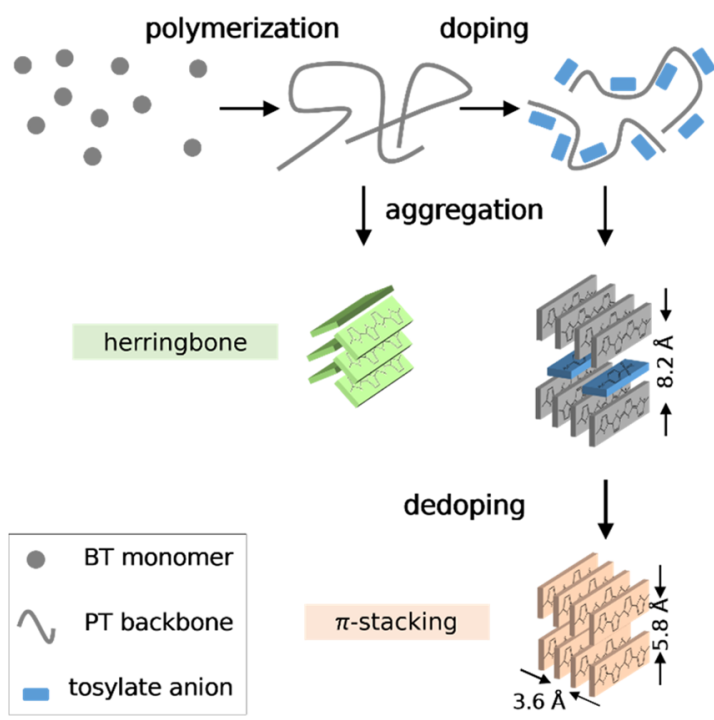

Figure 4. Schematic representation of the in situ polymerization and crystal structures present in PT films synthesized with FeTos. The two-step process of BT polymerization and subsequent doping with excess FeTos, which results in semiconducting PT chains aggregating in the herringbone motif, while highly doped PT arranges into $\pi$ stacked lamellae. Finally, dedoping of the lamellae results in a stable $\pi$ stacked, semiconducting PT crystal structure.

excess iron. Therefore, it is possible to control the resulting doping level by variation of the molar fraction of FeTos in the reaction mixture. Beyond a critical doping level, the doped polythiophene chains form cationic $\pi$-stacks. In the final doped films obtained after acetonitrile rinsing, these are arranged in a layered structure with layers of the tosylate counter ions in between. Opposite to that, the undoped chains aggregate in their natural $\mathrm{HB}$ motif. After dedoping and removal of the tosylate ions from the highly doped chains, the PT chains originally in the lamellar configuration retain their $\pi$-stacking motif, despite it usually not being their equilibrium crystal structure.

In summary, we presented a solution-based in situ polymerization process for unsubstituted semiconducting polymers that offers the possibility to exploit the involved doping process for influencing the type of crystal structure and aggregation of the polymer chains. We show that upon variation of the amount of oxidizing agent in the reaction mixture, the level of doping of the resulting films can be tuned. Aside from an increase in conductivity of the resulting conducting PT films, we are also able to show that an increased doping level has a sustainable impact on the structure. The GIWAXS measurements confirm the formation of a $\pi$-stacked lamellar structure beyond a critical level of doping. This $\pi$-stacking of the PT backbones is preserved in the crystallites even after dedoping and removal of the tosylate counter ions. This results in a new crystal structure of unsubstituted semiconducting polythiophene and, as shown by the spectroscopic analysis, in an increased intermolecular interaction. Here, native polythiophene (PT) serves as a model polymer for this process, which should be transferable to other conjugated polymers of similar chemical architecture.

\section{METHODS}

Thin Film Preparation. The in situ polymerization was performed by spin coating the metastable reaction mixture of 2,2'-bithiophene (Alfa Aesar) and iron(III) p-toluenesulfonate hexahydrate (Aldrich) in 1-propanol (synthesis grade, Roth). The precleaned glass substrates were coated with thin layers of polypropylene (Aldrich) by spin coating from 1-chlorobenzene (synthesis grade, Roth) to increase the adhesion of the polythiophene films during the posttreatment steps. All chemicals involved were used without further purification. The metastable reaction mixture was spin coated with 2000 $\mathrm{rpm}$ for $60 \mathrm{~s}$ at a substrate temperature of $90{ }^{\circ} \mathrm{C}$. Afterwards, the films were rinsed in either acetonitrile (doped polythiophene films) or ethanol (undoped polythiophene) for 30 min. Finally, the undoped PT films were annealed at $200{ }^{\circ} \mathrm{C}$ for $10 \mathrm{~min}$ in ambient atmosphere. For each experiment, the sum of the molar concentrations of PT and FeTos in the reaction mixture was kept constant upon variation of the ratio between them. Nevertheless, the overall concentration was set to match the resulting thickness required for each experimental method.

Four-Point Probe Measurements. The voltage was detected for a current sweep between 1.0 and $-1.0 \mu \mathrm{A}$, the sheet resistance was obtained by linear regression. The film thickness necessary for the conductivity calculation was determined by profilometry. The concentration of the reaction mixtures was $0.2 \mathrm{~mol} \mathrm{~L}^{-1}$.

Grazing Incidence Wide-Angle X-ray Scattering. The GIWAXS measurements were performed at the beamline 7.3.3 at the Advanced Light Source with an X-ray wavelength of $\lambda=$ $0.124 \mathrm{~nm}$ (energy of $10 \mathrm{keV}$ ). ${ }^{27}$ The scattered intensity was detected with a Pilatus $2 \mathrm{D}$ detector at a sample-to-detector distance of $299 \mathrm{~mm}$. The incident angle between the beam and the sample was $0.16^{\circ}$. The solid angle corrected $2 \mathrm{D}$ pattern as well as the radially integrated intensities were obtained with the GIXSGUI 1.6 software of Argonne National Laboratory, taking into account the necessary corrections. ${ }^{28}$ The concentration of the reaction mixtures was set to $0.7 \mathrm{~mol} \mathrm{~L}^{-1}$.

UV/vis Spectroscopy. The absorbance was obtained by performing transmission and reflection measurements with an integrating sphere. The luminescence spectra were obtained by spin coating onto polypropylene coated silicon substrates and measured in reflection. All emission spectra were measured with an excitation wavelength of $500 \mathrm{~nm}$. The concentration for both experiments was $0.2 \mathrm{~mol} \mathrm{~L}^{-1}$.

MD Simulations. Molecular dynamics simulations were performed with Gromacs using the Gromos 53a6 force field. $^{29,30}$ For the simulations, united-atom force field topologies were used, and the visual analysis of the molecular structure and trajectories was carried out with VMD. ${ }^{31}$ The structure file for the model of a PT 10-mer was generated with $\mathrm{JME},{ }^{32}$ based on which a force field file was created using the Automated Force Field Topology Builder and Repository. ${ }^{33}$ We enhanced the model by calculating partial charge distribution and the potential energy surface (PES) of the dihedrals between individual thiophene rings ourselves with Gaussian 09, rev. E.01. ${ }^{34}$ We used the B3LYP/6-31g(d,p) level for theory in all quantum chemical calculations. Tight convergence criteria were requested in geometry optimization. We utilized the symmetry of the molecule, so that the PES for the first dihedral was also used for the last and so on. We adjusted the model used for MD simulations to replicate the PES calculated by the quantum chemical calculations by rotating the molecule around the angle in question with enforced rotation. The potential energy of this process was determined with the Gromacs tool gmx energy. We implemented the new dihedrals with Ryckaert-Bellemans potentials. ${ }^{35}$ Simulations were performed locally and on the 
Jureca Supercomputer. ${ }^{36}$ Further information on the model system is given in the Supporting Information.

\section{ASSOCIATED CONTENT}

\section{S Supporting Information}

The Supporting Information is available free of charge on the ACS Publications website at DOI: 10.1021/acsomega.8b00684.

Absorbance spectra of doped polythiophene films; description and results of MD simulations (PDF)

\section{AUTHOR INFORMATION}

\section{Corresponding Author}

*E-mail: eva.herzig@uni-bayreuth.de.

\section{ORCID}

Stephan Gekle: 0000-0001-5597-1160

Eva M. Herzig: 0000-0002-0151-5562

Notes

The authors declare no competing financial interest.

\section{ACKNOWLEDGMENTS}

Financial support by the Bavarian State Ministry of Education, Science and the Arts via the project "Energy Valley Bavaria", the Deutsche Forschungsgemeinschaft (DFG) through TUM International Graduate School of Science and Engineering (IGSSE), and $\mathrm{BaCaTec}$ is acknowledged. Portions of this research were carried out at beamline 7.3.3 of the Advanced Light Source, which is supported by the Director of the Office of Science, Office of Basic Energy Sciences, of the U.S. Department of Energy under Contract No. DE-AC0205CH11231. The authors gratefully acknowledge the computing time granted by the John von Neumann Institute for Computing (NIC) and provided on the supercomputer JURECA at Jülich Supercomputing Center (JSC). A.B. and S.G. gratefully acknowledge funding from the Volkswagen Foundation and the Deutsche Forschungsgemeinschaft via Graduiertenkolleg 1640. This publication was funded by the German Research Foundation (DFG) and the University of Bayreuth in the funding programme Open Access Publishing.

\section{REFERENCES}

(1) Thompson, B. C.; Fréchet, J. M. J. Polymer-Fullerene Composite Solar Cells. Angew. Chem., Int. Ed. 2008, 47, 58-77.

(2) Arias, A. C.; MacKenzie, J. D.; McCulloch, I.; Rivnay, J.; Salleo, A. Materials and Applications for Large Area Electronics: Solution-Based Approaches. Chem. Rev. 2010, 110, 3-24.

(3) Rogers, J. A.; Someya, T.; Huang, Y. Materials and Mechanics for Stretchable Electronics. Science 2010, 327, 1603-1607.

(4) Sirringhaus, H.; Brown, P. J.; Friend, R. H.; Nielsen, M. M.; Bechgaard, K.; Langeveld-Voss, B. M. W.; Spiering, A. J. H.; Janssen, R. A. J.; Meijer, E. W.; Herwig, P.; de Leeuw, D. M.; et al. TwoDimensional Charge Transport in Self-Organized, High-Mobility Conjugated Polymers. Nature 1999, 401, 685-688.

(5) Singh, C. R.; Gupta, G.; Lohwasser, R.; Engmann, S.; Balko, J.; Thelakkat, M.; Thurn-Albrecht, T.; Hoppe, H. Correlation of Charge Transport with Structural Order in Highly Ordered Melt-Crystallized poly(3-Hexylthiophene) Thin Films. J. Polym. Sci., Part B: Polym. Phys. 2013, 51, 943-951.

(6) Bubnova, O.; Khan, Z. U.; Malti, A.; Braun, S.; Fahlman, M.; Berggren, M.; Crispin, X. Optimization of the Thermoelectric Figure of Merit in the Conducting Polymer Poly (3, 4-Ethylenedioxythiophene). Nat. Mater. 2011, 10, 429-433.
(7) Bravo-Grimaldo, E.; Hachey, S.; Cameron, C. G.; Freund, M. S. Metastable Reaction Mixtures for the in Situ Polymerization of Conducting Polymers. Macromolecules 2007, 7166-7170.

(8) McFarlane, S. L.; Deore, B. A.; Svenda, N.; Freund, M. S. A OneStep, Organic-Solvent Processable Synthesis of PEDOT Thin Films via in Situ Metastable Chemical Polymerization. Macromolecules 2010, 43, 10241-10245.

(9) Elschner, A. From EDOT to PEDOT Oxidative Polymerization and Other Routes. In PEDOT - Principles and Applications of an Intrinsically Conductive Polymer; Taylor and Francis Group, 2011; pp 67-82.

(10) Chaalane, A.; Mahi, D.; Dkhissi, A. Structural and Electronic Properties of Doped Oligothiophenes in the Presence of PToluenesulfonate Acids. Theor. Chem. Acc. 2015, 134, No. 66.

(11) Borrelli, D. C.; Barr, M. C.; Bulović, V.; Gleason, K. K. Bilayer Heterojunction Polymer Solar Cells Using Unsubstituted Polythiophene via Oxidative Chemical Vapor Deposition. Sol. Energy Mater. Sol. Cells 2012, 99, 190-196.

(12) Borrelli, D. C.; Lee, S.; Gleason, K. K. Optoelectronic Properties of Polythiophene Thin Films and Organic TFTs Fabricated by Oxidative Chemical Vapor Deposition. J. Mater. Chem. C 2014, 2, 7223.

(13) Kaneto, K.; Yoshino, K.; Inuishi, Y. Electrical and Optical Properties of Polythiophene Prepared by Electrochemical Polymerization. Solid State Commun. 1983, 46, 389-391.

(14) Jeon, S. S.; Yang, S. J.; Lee, K. J.; Im, S. S. A Facile and Rapid Synthesis of Unsubstituted Polythiophene with High Electrical Conductivity Using Binary Organic Solvents. Polymer 2010, 51, 4069-4076.

(15) Li, X. G.; Li, J.; Meng, Q. K.; Huang, M. R. Interfacial Synthesis and Widely Controllable Conductivity of Polythiophene Microparticles. J. Phys. Chem. B 2009, 113, 9718-9727.

(16) Yamamoto, T.; Morita, A.; Miyazaki, Y.; Maruyama, T.; Wakayama, H.; Zhou, Z.-H.; Nakamura, Y.; Kanbara, T.; Sasaki, S.; Kubota, K. Preparation of .Ir-Conjugated Poly(thiophene-2,5-Diyl), Poly@-Phenylene), and Related Polymers Using Zerovalent Nickel Complexes. Linear Structure and Properties of the 7-Conjugated Polymers. Macromolecules 1992, 25, 1214-1223.

(17) Miller, L. L.; Mann, K. R. $\pi$-Dimers and $\pi$-Stacks in Solution and in Conducting Polymers. Acc. Chem. Res. 1996, 29, 417-423.

(18) Brocks, G. П-Dimers of Oligothiophene Cations. J. Chem. Phys. 2000, 112, 5353-5363.

(19) Brückner, S.; Porzio, W. The Structure of Neutral Polythiophene. An Application of the Rietveld Method. Makromol. Chem. 1988, 189, 961-967.

(20) Mo, Z.; Lee, K. B.; Moon, Y. B.; Kobayashi, M.; Heeger, A. J; Wudl, F. X-Ray Scattering from Polythiophene: Crystallinity and Crystallographic Structure. Macromolecules 1985, 18, 1972-1977.

(21) Youm, S. G.; Hwang, E.; Chavez, C. A.; Li, X.; Chatterjee, S.; Lusker, K. L.; Lu, L.; Strzalka, J.; Ankner, J. F.; Losovyj, Y.; et al. Polythiophene Thin Films by Surface-Initiated Polymerization: Mechanistic and Structural Studies. Chem. Mater. 2016, 28, 47874804.

(22) Prosa, T. J.; Winokur, M. J.; Moulton, J.; Smith, P.; Heeger, A. J X-Ray Structural Studies of Poly(3-Alkylthiophenes): An Example of An Inverse Comb. Macromolecules 1992, 25, 4364-4372.

(23) Aasmundtveit, K. E.; Samuelsent, E. J.; Pettersson, L. A. A.; Inganäs, O.; Johansson, T.; Feidenhans, R. Structure of Thin Films of Poly (3, 4-Ethylenedioxythiophene). Synth. Met. 1999, 101, 561-564.

(24) Panzer, F.; Bässler, H.; Köhler, A. Temperature Induced OrderDisorder Transition in Solutions of Conjugated Polymers Probed by Optical Spectroscopy. J. Phys. Chem. Lett. 2017, 8, 114-125.

(25) Spano, F. C. Modeling Disorder in Polymer Aggregates: The Optical Spectroscopy of Regioregular poly(3-Hexylthiophene) Thin Films. J. Chem. Phys. 2005, 122, No. 234701.

(26) Spano, F. C.; Silva, C. H- and J-Aggregate Behavior in Polymeric Semiconductors. Annu. Rev. Phys. Chem. 2014, 65, 477-500.

(27) Hexemer, A.; Bras, W.; Glossinger, J.; Schaible, E.; Gann, E.; Kirian, R.; MacDowell, A.; Church, M.; Rude, B.; Padmore, H. A 
SAXS/WAXS/GISAXS Beamline with Multilayer Monochromator. J. Phys.: Conf. Ser. 2010, 247, No. 012007.

(28) Jiang, Z. GIXSGUI: A MATLAB Toolbox for Grazing-Incidence $\mathrm{X}$-Ray Scattering Data Visualization and Reduction, and Indexing of Buried Three-Dimensional Periodic Nanostructured Films. J. Appl. Crystallogr. 2015, 48, 917-926.

(29) Hess, B.; Kutzner, C.; van der Spoel, D.; Lindahl, E. GROMACS 4: Algorithms for Highly Efficient, Load-Balanced, and Scalable Molecular Simulation. J. Chem. Theory Comput. 2008, 4, 435-447.

(30) Oostenbrink, C.; Villa, A.; Mark, A. E.; Van Gunsteren, W. F. A Biomolecular Force Field Based on the Free Enthalpy of Hydration and Solvation: The GROMOS Force-Field Parameter Sets 53A5 and 53A6. J. Comput. Chem. 2004, 25, 1656-1676.

(31) Humphrey, W.; Dalke, A.; Schulten, K. VMW: Visual Molecular Dynamics. J. Mol. Graphics 1996, 14, 33-38.

(32) Ertl, P. Molecular Structure Input on the Web. J. Cheminf. 2010, $2,1$.

(33) Malde, A. K.; Zuo, L.; Breeze, M.; Stroet, M.; Poger, D.; Nair, P. C.; Oostenbrink, C.; Mark, A. E. An Automated Force Field Topology Builder (ATB) and Repository: Version 1.0. J. Chem. Theory Comput. 2011, 7, 4026-4037.

(34) Frisch, M. J.; Trucks, G. W.; Schlegel, H. B.; Scuseria, G. E.; Robb, M. A.; Cheeseman, J. R.; Scalmani, G.; Barone, V.; Mennucci, B.; Petersson, G. A.; Nakatsuji, H.; Caricato, M.; Li, X.; Hratchian, H. P.; Izmaylov, A. F.; Bloino, J.; Zheng, G.; Sonnenberg, J. L.; Hada, M.; Ehara, M.; Toyota, K.; Fukuda, R.; Hasegawa, J.; Ishida, M.; Nakajima, T.; Honda, Y.; Kitao, O.; Nakai, H.; Vreven, T.; Montgomery, J. A., Jr.; Peralta, J. E.; Ogliaro, F.; Bearpark, M.; Heyd, J. J.; Brothers, E.; Kudin, K. N.; Staroverov, V. N.; Keith, T.; Kobayashi, R.; Normand, J.; Raghavachari, K.; Rendell, A.; Burant, J. C.; Iyengar, S. S.; Tomasi, J.; Cossi, M.; Rega, N.; Millam, J. M.; Klene, M.; Knox, J. E.; Cross, J. B.; Bakken, V.; Adamo, C.; Jaramillo, J.; Gomperts, R.; Stratmann, R. E.; Yazyev, O.; Austin, A. J.; Cammi, R.; Pomelli, C.; Ochterski, J. W.; Martin, R. L.; Morokuma, K.; Zakrzewski, V. G.; Voth, G. A.; Salvador, P.; Dannenberg, J. J.; Dapprich, S.; Daniels, A. D.; Farkas, O.; Foresman, J. B.; Ortiz, J. V.; Cioslowski, J.; Fox, D. J. Gaussian 09, revision E.01; Gaussian, Inc.: Wallingford, CT, 2013.

(35) Abraham, M.; Hess, B.; van der Spoel, D.; Lindahl, E. GROMACS Reference Manual, version 2016.3, 2016.

(36) Jülich Supercomputing Centre. JURECA: General-Purpose Supercomputer at Jülich Supercomputing Centre. JLSRF 2016, 2, A62. 